REVISTA DE LA FACULTAD DE CONTADURÍA PÚBLICA | ACTIVOS

\title{
Algunos nuevos retos de la información contable-financiera: intangibles, Internet y gestión de beneficios
}

Leandro Cañibano Calvo ${ }^{1}$

Cañibano C., L. (2007). Algunos nuevos retos de la información contable-financiera: intangibles, Internet y gestión de beneficios. Revista Activos, 13, 89-115.

Recibido: 25 de marzo 2007 Aprobado: 29 de noviembre 2007

\section{Resumen}

El desarrollo de la economía, basada en el conocimiento, ha significado cambios tecnológicos, económicos y sociales que caracterizan el quehacer de las organizaciones hoy día. Esto ha afectado profundamente el contenido, la difusión y la estrategia de preparación de la información contable-financiera de las organizaciones. Aspectos como los intangibles, la divulgación de información a través del Internet y la gestión contable de beneficios, se han vuelto significativos en los reportes financieros. De allí que de lo que se trata es de nuevas formas de reconocimiento y valoración de los recursos intangibles, así como nuevos lenguajes para la transmisión de datos contables como es el XBRL y la generación de información contable sustentada en premisas de transparencia y mejor información.

Palabras clave: Valoración de intangibles, Extensible Business Reporting Language (XBRL), Normas internacionales para informes financieros (NIIF), Organización para la Cooperación y el Desarrollo Económico (OCDE). JEL: G14, M41.

1 Licenciado en Ciencias Económicas y Doctor en Ciencias Económicas de la Universidad Complutense de Madrid. Catedrático de Economia Financiera y Contabilidad de la Universidad Autónoma de Madrid, leandro. canibano@uam.es. 
ACTIVOS | REVISTA DE LA FACULTAD DE CONTADURÍA PÚBLICA

Cañibano C., L. (2007). Some new challenges of the accounting-financial information: intangible, internet and management of benefits. Activos Review, 13, 89-115.

\begin{abstract}
The development of the economy based on the knowledge has meant technological, economic and social changes that characterize the chore of the organizations today. This has affected deeply the content, the diffusion and the strategy of preparation of the countablefinancial information of the organizations. Aspects like the intangible ones, the information publication across the Internet and the book-keeping of benefits, have become significant in the financial reports. From there it is a question that performs new forms of recognition and evaluation of the intangible resources, as well as new languages for the broadcast of countable information as it is the XBRL and the generation of countable information sustained in premises of transparence and better information
\end{abstract}

Key words: Evaluation of Intangible, Extensible Business Reporting Language (XBRL), international Norms for financial reports (NIIF), Organization for the Cooperation and the Economic Development (OCDE). JEL: G14, M41.

Cañibano C., L. (2007). Quelques nouveaux défis de l'information comptable - financière : intangibles, internet et gestion de bénéfices. Revue Activos, 13, 89-115.

\title{
Résumé
}

Le développement de l'économie basée sur la connaissance a apporté des grandes transformations technologiques, économiques et sociales caractérisant le travail des organismes aujourd'hui. Cela a affecté profondément le contenu, la diffusion et la stratégie de préparation de l'information comptable et financière des organismes. Les concepts comme l'intangible, la divulgation d'information à travers l'internet et la gestion comptable de bénéfices, sont devenus significatifs dans les rapports financiers. Il s'agit des nouvelles formes de reconnaissance et la valorisation des ressources intangibles, ainsi que de nouveaux langages pour la transmission des données comptables comme celui du XBRL et la gestion de l'information comptable soutenue dans des prémisses basées sur la transparence et la meilleure information.

Mots clé: La valorisation des Intangibles, Extensible Business Reporting Language (XBRL), Normes internationaux pour les rapports financiers (NIIF), Organisme pour la Coopération et le Développement Économique (OCDE). JEL: G14, M41. 


\section{Introducción}

Durante la pasada década de los noventa, el proceso de regulación y armonización contable internacional ha experimentado grandes adelantos, en particular en el ámbito de la Unión Europea (UE), donde la estrategia en pro de la adopción de las Normas Internacionales de Contabilidad se ha visto culminada con la promulgación en 2002 y 2003 de los Reglamentos del Consejo y el Parlamento Europeo y de la Comisión Europea, lo que significa que, a partir de 2005, las cuentas consolidadas de las sociedades cotizadas en bolsas radicadas en la UE, deberán ser formuladas de acuerdo con las indicadas Normas Internacionales de Contabilidad. Con ello, la declarada equivalencia y comparabilidad de las cuentas anuales de las compañías europeas puede llegar a convertirse en una realidad.

La información contable-financiera de las sociedades ha pasado a ser considerada como un bien jurídicamente protegible, dada la utilidad de la misma para un amplio conjunto de usuarios, entre los que se incluyen los accionistas, los empleados, los acreedores y otros terceros interesados en la marcha de la empresa. Las autoridades de la Unión Europea que establecieron como objetivo la libre circulación de capitales en el mercado interior consideraron que para el cumplimiento de dicho objetivo, la protección de los inversores y el mantenimiento de la confianza de los mercados financieros, era necesario profundizar en el proceso de armonización contable europea, yendo más allá de las Directivas de derecho de sociedades y adoptando las Normas Internacionales de Contabilidad o Normas Internacionales de Información Financiera, de manera tal que los estados financieros consolidados de las sociedades cotizadas en bolsa resulten comparables.

Pero, al mismo tiempo que se ha progresado en la dirección que acabamos de apuntar, se han producido también importantes cambios tecnológicos, económicos y sociales que afectan profundamente al contenido, a la difusión y a la estrategia de preparación de la información contable-financiera. Este trabajo trata sobre tres de ellos que consideramos altamente relevantes: intangibles, Internet y gestión contable de beneficios, pero nos gustaría dejar constancia que además existen algunos otros, también de gran entidad².

El desarrollo de la Economía basada en el conocimiento ha puesto de manifiesto la importancia de los intangibles como elementos generadores de valor en la empresa, sin que la información contable-financiera de las compañías ofrezca detalle puntual de esta nueva

2 Las ideas expuestas en este trabajo forman parte del contenido del Discurso sobre "Información Financiera y Gobierno de la Empresa", pronunciado por el autor en su toma de posesión como Académico Numerario de la Real Academia de Doctores. Madrid, 21 de enero de 2003.

ISSN: $0124-5805-$ No. 13 - 2007 - pp. 89-115 | 91 
ACTIVOS | REVISTA DE LA FACULTAD DE CONTADURÍA PÚBLICA

realidad, más importante en muchos casos que la relativa a los elementos tangibles, sobre los que sí ofrece en cambio datos suficientes.

Uno de los pilares de esa nueva Economía basada en el conocimiento son las tecnologías de la información y las comunicaciones, mediante las que la difusión de la información financiera está alcanzando cotas difíciles de imaginar hace muy pocos años, dada la gran fluidez de aplicaciones lograda a lo largo de la cadena del suministro de información. La dirección de las empresas ha incorporado las variables contables a su estrategia, procediendo a realizar una gestión contable de beneficios, en aras de cumplir sus objetivos o de alcanzar los beneficios esperados por el mercado, lo cual ha desembocado lamentablemente en algunos de los más sonados escándalos empresariales.

Estos son los temas que trataremos a continuación, dedicando a cada uno de ellos sucesivos apartados, sin perjuicio de reconocer que también otros de importancia podrían ser considerados como actuales retos para la información financiera, como por ejemplo los relativos a la información sobre riesgos y la información sobre responsabilidad social corporativa.

\section{La relevancia de los intangibles}

Uno de los grandes retos de investigación en la actualidad es el de cómo hacer frente a las transformaciones que se observan en el entorno económico. Estamos abandonando la era industrial para adentrarnos en la era del conocimiento, y este cambio tiene importantes implicaciones para los sistemas de valoración y gestión de empresas.

Si hace tan sólo dos décadas, el valor de una organización se asimilaba fundamentalmente al valor de sus recursos tangibles y financieros, en la actualidad, el potencial de desarrollo futuro de las empresas descansa, principalmente, sobre sus recursos intangibles. Los sistemas contables en vigor fueron diseñados durante la era industrial, y responden a la necesidad de valorar los recursos tangibles de la empresa, pero resultan muy limitados en la nueva economía del conocimiento. Esta limitación de los sistemas contables para capturar el valor de las organizaciones se traduce en una mayor volatilidad e ineficiencia de los mercados de capitales (que no disponen de información fiable sobre las verdaderas fuentes de creación de valor de las sociedades cotizadas), así como también en una gestión ineficaz de las capacidades de la empresa. 


\section{Creciente importancia de los intangibles}

El creciente peso de los intangibles, demanda nuevas formas de valoración de la empresa, nuevos instrumentos para su medición y comunicación, y también nuevas formas de valoración de la economía en su conjunto. Fruto de esta necesidad, la década de los noventa del pasado siglo ha sido testigo de un creciente número de iniciativas en el ámbito internacional sobre la medición y gestión de los intangibles a nivel macro, meso y microeconómico (Chaminade \& Cañibano, 2003).

La primera llamada de atención sobre la creciente importancia de los intangibles en la Economía surgió en el seno de la Organización para la Cooperación y el Desarrollo Económico (OCDE) en la pasada década de los ochenta. Utilizando datos agregados, la OCDE demostró que las inversiones en intangibles tales como la educación, I+D, o software estaban aumentando a un ritmo mucho más rápido que las inversiones en elementos tangibles (OCDE, 1997b) y que, por tanto, de continuar dicha tendencia, la economía iba a estar crecientemente basada en el conocimiento.

Los estudios pioneros de la OCDE abrieron la puerta a un conjunto muy relevante de iniciativas internacionales sobre la valoración de los intangibles y su impacto en el crecimiento (Johanson 2002), entre las que destacan varias conferencias organizadas por la propia OCDE (OECD 1997a, 1997b, 1999) y la Unión Europea (Cañibano \& Sánchez, 2003), un número creciente de publicaciones sobre la valoración de los intangibles entre las que sobresalen diversos artículos de revisión de la literatura (Cañibano et al., 1999 y 2000, Johanson et al., 1999, Petty \& Guthrie, 2000) y diversos proyectos internacionales en los que vienen participando distintas empresas e investigadores.

Dentro de este último apartado, se puede afirmar que Europa ha liderado la investigación en medición, gestión y difusión de información sobre intangibles. Aunque en Estados Unidos se han llevado a cabo algunos proyectos de relevancia ${ }^{3}$, ha sido en Europa donde se han desarrollado los proyectos de mayor envergadura e impacto internacional, muchos de ellos con el apoyo financiero de la Unión Europea. También es en Europa donde se encuentran las empresas pioneras en el desarrollo de herramientas para la gestión del capital intelectual o los intangibles, fundamentalmente en los países nórdicos. intangibles (Conference Board) y, el segundo, sobre la Relación entre los intangibles y el valor de la empresa en la Nueva Economia (Brooking Institution Task Force on Understanding Intangibles) (Blair y Wallman 2001), así como los numerosos estudios liderados por el profesor Baruch Lev de la New York University (http://www.baruch-lev.com). 
ACTIVOS | REVISTA DE LA FACULTAD DE CONTADURÍA PÚBLICA

Uno de los primeros proyectos desarrollados en Europa, centrado en el análisis de los intangibles, ha sido el proyecto Meritum (1998-2001) (Measuring Intangibles to Understand and Improve Innovation Management), coordinado por el grupo de la Universidad Autónoma de Madrid bajo la responsabilidad del autor de estas líneas, y en el que participaron nueve instituciones de seis países europeos. Meritum ha continuado, a partir de 2001, a través de la red temática $E^{\star}$ KNOW-NET, financiada por la Unión Europea y centrada, también, en la gestión y valoración de intangibles.

En el año 2000, y ante el creciente interés que estos temas estaban adquiriendo a nivel internacional, la Unión Europea financió la creación de un Grupo de Expertos en Intangibles (High Level Expert Group) a quienes les encargó la elaboración de un Informe sobre la Economía Intangible y su impacto (Unión Europea, 2003). En el año 2000 también se puso en marcha un tercer proyecto europeo: RESCUE/PRISM cuyo objetivo fundamental consistió en la elaboración de estudios de caso sobre gestión de intangibles que pudieran utilizarse con fines de formación.

Todas estas iniciativas han producido como resultado numerosas evidencias sobre el creciente papel que tienen los intangibles en los procesos de generación de valor de las empresas, tanto a nivel macro-económico (Cuentas nacionales), como a nivel mesoeconómico (Mercados de capitales) y a nivel micro-económico (organización).

En el ámbito macroeconómico, la OCDE ha estimado el peso de la participación en el PIB de un conjunto de países, de intangibles, tales como la educación, la I+D o el software. Vickery (1999) demuestra que la inversión en activos intangibles ${ }^{4}$ en los países de la OCDE representa un $10 \%$ del PIB, manteniendo un ritmo de crecimiento muy superior al de los activos tangibles.

Desde un punto de vista mesoeconómico existe un gran número de trabajos que estiman el impacto de los intangibles en los mercados de capitales, incidiendo, como señala García-Ayuso (2003), en aspectos muy diversos, tales como: los efectos negativos de la escasa información sobre intangibles, sobre el riesgo y el coste del capital, la habilidad de las empresas para obtener capital adicional y el impacto de los intangibles sobre algunas variables contables fundamentales. Otros tratan sobre el análisis de la responsabilidad social corporativa (Guthrie et al., 2001, Holland \& Johanson 2003); los mecanismos de difusión de la información sobre intangibles (Bukh et al., 2002) y su interpretación por analistas

Sólo se consideran las inversiones en educación, I+D y software, es decir, aquellos intangibles para los que se dispone de información agregada. 
financieros (Roberts, 2002); y, finalmente, un grupo de estudios dirigidos al desarrollo de métodos para la estimación del valor de los intangibles, basados en el modelo de valoración de Ohlson (1995), que relacionan dichos intangibles con el resultado contable y el valor de mercado de las acciones (Giner \& Pardo, 2002, Ballester et al., 2003, Giner \& Rees, 2001, García \& Mora, 2003a y 2003b).

Dentro de este nivel mesoeconómico, también se encuentran algunos trabajos sobre capital social e intangibles o sobre el impacto de la cultura nacional en la gestión de los intangibles que demuestran el impacto del entorno social y cultural respectivamente, en la gestión de los intangibles (Chaminade \& Johanson, 2003).

Finalmente, en el ámbito empresarial, la gran mayoría de esfuerzos se han centrado en el análisis de los procesos de gestión de conocimiento (Sánchez et al., 2000, Johanson, 2000, Johanson et al., 2001b, Roberts \& Chaminade, 2003), la medición de intangibles (Cañibano et al., 2002), el impacto de la gestión de los intangibles en el desempeño de la empresa (Johanson et al., 2001a), o el desarrollo de Informes de Capital Intelectual (Mouritsen et al., 2001a y 2001b, Danish Agency for Trade and Industry, 2002, Cañibano et al., 2002, Danish Ministry of Science and Technology, 2003a y 2003b).

De forma simplificada, los trabajos anteriormente mencionados se podrían clasificar en dos grandes grupos: uno que atiende a la valoración de los intangibles como instrumento de gestión interna y otro que orienta dicha valoración como herramienta de comunicación externa. El primero está directamente relacionado con el aprendizaje organizativo, con la organización inteligente o basada en el conocimiento; el segundo, está relacionado con la valoración externa de la empresa, con la información que ésta suministra a sus accionistas y otros terceros interesados en la misma, incluidos, por supuesto, los mercados de capitales.

\section{Información financiera y no - financiera sobre intangibles}

En relación con la información externa sobre intangibles dirigida a terceros, mencionada en el epígrafe precedente, cabe señalar, que la información contable-financiera tradicionalmente ha venido incluyendo en los balances los valores de ciertos intangibles calificados por Hendriksen y Van Breda (1992) como intangibles tradicionales (marcas, patentes, nombres comerciales, derechos de autor, etc.); sin embargo, no ocurre así con los denominados nuevos intangibles (capacidad para atraer y retener recursos humanos, fidelidad de la clientela, capacidad innovadora, flexibilidad de la organización, etc.), salvo cuando se produce una venta de la empresa, en cuyo caso el precio global pagado por ella considera 
ACTIVOS | REVISTA DE LA FACULTAD DE CONTADURÍA PÚBLICA

todos los activos netos adquiridos, estén o no contabilizados, entre los que lógicamente se encuentran los nuevos intangibles comentados.

El exceso del precio pagado sobre el valor real de los activos tangibles e intangibles tradicionales adquiridos es lo que constituye el Fondo de Comercio, el cual es reconocido contablemente como un activo más del comprador, cuya sustancia no es otra cosa que los intangibles susceptibles de generar valor, que no habían sido objeto de reconocimiento contable en la empresa adquirida.

De acuerdo con lo anterior, cuando una empresa adquiere a otra empresa en funcionamiento, la primera de ambas puede incluir entre sus activos el valor de los intangibles por los que ha satisfecho un precio, pero no puede hacerlo con los intangibles internamente generados por ella misma, porque tales intangibles no han sido objeto de transacción alguna. Así pues, la información contable-financiera de dicha empresa no valora todos sus elementos intangibles de la misma manera, unos -los correspondientes a la empresa adquirida- se encontrarán incluidos en su balance, pero otros -los internamente generados- no lo estarán, lo que da lugar a una valoración asimétrica de los intangibles y a una información poco útil en términos comparativos, de la propia empresa en el tiempo y en relación con otras empresas.

Algunos autores han defendido la tesis de que los activos intangibles deberían recibir un tratamiento contable análogo a los activos tangibles, Wallman (1995) pone de manifiesto que:

Algunos de los nuevos y más significativos valores de negocio apenas aparecen en los balances, los cuales ofrecen una pobre imagen de la verdadera posición financiera de las empresas. Por ejemplo, laboratorios como Merck no muestran activo alguno relacionado con sus innovaciones de productos, ... la marca Coca-Cola no se refleja como un activo en el balance de dicha compañía,... Microsoft cuyas acciones valen decenas de billones de dólares presenta un balance que le hace aparecer mucho más pequeña (p. 85).

Lev y Zarowin (1998), por su parte, indican que:

Esta política contable acabaría con el absurdo de que los ladrillos y el mortero de las empresas químicas, farmacéuticas, electrónicas, informáticas, de biotecnología y de 
telecomunicaciones sean consideradas como activos, mientras que las inversiones en intangibles generadoras de la mayoría de sus ingresos no pueden encontrarse en sus estados financieros (p. 363).

La tesis anterior ha estado soportada por diversos estudios, inicialmente, los realizados por Lev (1996, 1998 y 2001), en los que se ponía de manifiesto el paulatino alejamiento de los valores contables de las empresas de sus respectivos valores de cotización en el mercado bursátil. Este alejamiento no se trata de una circunstancia temporal, sino de una tendencia observada durante más de veinte años, que da lugar a que la Bolsa valore las acciones multiplicando por cuatro y hasta por seis su valor contable 5 . Esto significa que se valora mucho más lo que no aparece en el balance -los intangibles- que lo que se muestra en él.

Pero, el armazón de los principios de contabilidad generalmente aceptados se encuentra fuertemente cohesionado, no ha resultando fácil introducir cambios radicales en él, por ello, el tratamiento contable análogo de activos tangibles e intangibles todavía deberá esperar. No obstante, algún cambio de importancia sí que ha llegado a producirse, de momento en los US GAAP (FASB, 2001) y, según los indicios, también se producirá a corto plazo en las NIC/NIIF.

Dicho cambio atañe al tratamiento contable del Fondo de Comercio, que ha pasado a ser considerado como un activo no amortizable $e^{6}$, por lo que no procede su imputación sistemática a los resultados del ejercicio durante un plazo convencional ${ }^{7}$; procede en cambio la realización de un test de deterioro, ejercicio tras ejercicio, para comprobar si se mantiene o no el valor contable del mismo, dependiendo lo anterior de las expectativas de beneficios futuros del negocio correspondiente. Comprobada la existencia de un deterioro de dicho valor, su importe deberá ser contabilizado de inmediato en su totalidad, con el consiguiente reconocimiento de la pérdida como resultado del ejercicio ${ }^{8}$.

5 El ratio medio Precio/ Valor contable (Market to Book) de las 500 empresas incluidas en el indice Standard \& Poor, cotizadas en la Bolsa de Nueva York, a 31 de diciembre de 2000 era de 6,3 (Lev 2001). A 31 de marzo de 2002 era de 4,3 (Di Piazza et al., 2002, p.40). En España dicho ratio era de 2,2 (Cañibano et al., 2000b), siendo destacable el ratio M/B correspondiente a algunas empresas a 31 de diciembre de 2003, como Telefónica móviles: 10,8 o Inditex: 9,7.

$6 \quad$ Una de las características de los intangibles que forman parte del Fondo de Comercio consiste en que los mismos no se deprecian, pueden ser utilizados de manera simultánea y repetitiva sin que disminuya su utilidad, de ahi su no-amortización.

7 Veinte años como máximo en España, de acuerdo con la NIC vigente.

8 La contabilización del deterioro del Fondo de Comercio, según la regulación contable norteamericana, dio lugar a que los resultados de algunas compañías españolas cotizadas en la Bolsa de Nueva York (SCH, BBVA, Telefónica) se vieran sensiblemente reducidos en el ejercicio 2001. 
ACTIVOS | REVISTA DE LA FACULTAD DE CONTADURÍA PÚBLICA

Con esta incorporación al balance de algunos intangibles, vía Fondo de Comercio no amortizable, no se completa el reconocimiento contable de estos elementos, porque todavía quedan fuera de la información contable todos aquellos intangibles internamente generados que no han sido objeto de una transacción similar a la que originó el Fondo de Comercio, o que se corresponden con Fondos de Comercio ya amortizados, por haber surgido éstos con anterioridad a la aplicación de la norma que prevé su no amortización. Para subsanar esta carencia informativa, el regulador contable norteamericano puso en marcha en 2001 su proyecto sobre "Información a revelar sobre activos intangibles" (FASB, 2003), en el que se planteaba, no el reconocimiento en balance de los valores correspondientes, pero sí al menos, la inclusión de una información sobre los mismos en las notas de la memoria de los estados financieros.

El CNIC, en su declaración programática de diciembre de 2000, se plantea también la conveniencia de revelar información sobre intangibles, sin precisar todavía si esta información debiera formar parte de las notas a los estados financieros o de otra información de índole complementaria, mediante la que podrían suministrarse comentarios cualitativos de la dirección, predicciones de futuros flujos de caja o valoraciones de expertos independientes. Esta información complementaria de tipo narrativo constituiría una nueva línea o capa de información en el sentido apuntado por Wallman (1996).

Esta vía de información sobre los intangibles a través de un informe específico, con independencia del desarrollo que en el futuro pueda alcanzar la información que sobre los mismos contengan los estados financieros, ha sido objeto de especial atención en el ámbito de la Unión Europea, según ha quedado apuntado en el epígrafe precedente. "Directrices para la gestión y difusión de información sobre intangibles (Informe de Capital Intelectual)" (Cañibano et al., 2002) y "Directrices danesas" (Danish Ministry of Science and Technology, 2003a), ambas de naturaleza voluntaria, constituyen una buena guía mediante la que las empresas pueden revelar información sobre sus intangibles de manera sistemática y estructurada.

El Libro Blanco sobre la reforma contable en España, tras referirse a los intangibles como elementos fundamentales de la creación de valor en la empresa, sintetiza las partes componentes del "Informe de Capital Intelectual" contenido en el Proyecto Meritum (Gonzalo, 2002a), de la forma siguiente:

El "Informe de Capital Intelectual" consta de tres partes: Visión de la empresa, Resumen de recursos y actividades intangibles y Sistema de indicadores. 
- La Visión de la empresa describe en qué se diferencia la empresa de sus competidores y qué recursos intangibles le permiten marcar distancias con otras organizaciones. Se narra cómo los distintos agentes se benefician de las actividades dirigidas a generar conocimiento en la empresa, justificando, por ejemplo, el interés de invertir en la empresa u obtener un empleo en la misma.

- En el Resumen de recursos y actividades intangibles se describen los recursos intangibles de que puede disponer la empresa y las diferentes actividades llevadas a cabo hasta la fecha, y las que se realizarán en el futuro para incrementar el valor de dichos recursos. La presentación de los recursos y actividades intangibles se refiere a las tres categorías del capital intelectual: capital humano, capital estructural y capital relacional.La empresa debe mostrar que es precisa una combinación única de acciones para alcanzar los objetivos estratégicos y que la compañía está, en la práctica, desarrollando estas acciones.

- El Sistema de indicadores de los recursos y actividades intangibles, permite a los proveedores de bienes, servicios o capitales de la empresa, llevar a cabo una estimación acertada de los beneficios futuros de la empresa y del riesgo que conlleva la inversión en ella. Esta información será de mayor utilidad cuando no sólo se presenten los indicadores, sino también, su tendencia esperada y su relación con los resultados y el crecimiento futuros de la empresa (p.164).

Concluye el Libro Blanco recomendando al regulador la normalización y divulgación de los indicadores sobre intangibles, para su uso voluntario por parte de las empresas, aparte de considerar conveniente también, la eliminación de algunas de las restricciones que impiden la inclusión en balance de algunos intangibles.

\section{La aplicación de las nuevas tecnologías de la información y la comunicación}

Internet es una innovación que ha transformado profundamente los procesos de transmisión de información en la sociedad en general y en el mundo empresarial en particular. El punto clave de todas las ventajas que proporciona la red se encuentra en la "conectividad", en la posibilidad de ofrecer y demandar información en tiempo real y de satisfacer de inmediato las necesidades informativas, incentivando un nuevo tipo de comercio que se extiende por todo el mundo. "Se podría decir que el lema de Internet representa un sueño: el acceso de todos a toda la información en todo momento y en cualquier lugar" (Gandía, 2002, p.771). 
ACTIVOS | REVISTA DE LA FACULTAD DE CONTADURÍA PÚBliCA

La posibilidad de difundir la información contable-financiera por medio de Internet supone una nueva vía de comunicación entre las empresas y sus accionistas y otros terceros interesados en la marcha de las mismas, que puede llegar a arrumbar la tradicional información financiera de carácter histórico para ser sustituida por otra en tiempo real. De hecho, los directivos de las compañías ya utilizan información de esta naturaleza para basar en ella sus decisiones, por lo tanto es posible que, no tardando mucho, el tradicional modelo de rendición de cuentas anuales, tal como hoy lo conocemos, sufra profundas transformaciones, dado el desfase temporal que lleva consigo el mismo, a pesar de los estados financieros de información intermedia normalmente preparados, trimestral o semestralmente, por las compañías cotizadas en las bolsas de valores.

Pero antes de llegar a ese futurible que representa la posibilidad de acceder en tiempo real a la información contable-financiera de las empresas, veamos los pasos que se están dando para sacar partido a este nuevo vehículo de comunicación que es Internet, dentro del contexto de la información de índole histórica que, al poder ser difundida por los nuevos medios que proporciona la red, puede llegar con mayor facilidad a sus potenciales usuarios y puede ser ampliada sin las restricciones que siempre representa su presentación tradicional en papel. "El reporting financiero electrónico ha venido a democratizar y diseminar la información financiera, dado que su divulgación es oportuna, interactiva, cómoda, económica, frecuente, reutilizable y compartida por los usuarios, especialmente por los inversores" (Carreira, 2003, p. 257).

Según un estudio relativo a las 100 sociedades cotizadas más importantes de Europa (Bonsón, 2001), todas ellas tenían una página web activa y ofrecían en ésta el ejemplar completo de su informe financiero anual, que incluye las cuentas anuales y el informe de auditoría. Sólo 64 sociedades incluían los estados financieros intermedios, trimestrales o semestrales, sin auditar; 47 compañías suministraban un resumen de sus cuentas correspondientes a los últimos cinco años y 24 incluían ratios financieros. Por lo que se refiere a los Estados Unidos, todas las sociedades cotizadas en la Bolsa de Nueva York suministran en su web sus cuentas anuales completas o bien un enlace con los archivos de EDGAR, la base de datos electrónicos de la Comisión del Mercado de Valores de dicho país (SEC), donde se encuentran los informes contable-financieros de todas las sociedades cotizadas. Algunas de ellas ofrecen otra información adicional ${ }^{9}$, especialmente, noticias de la prensa financiera, pero por el momento no se encuentra disponible información en tiempo real, salvo la relativa a las cotizaciones. 
En España todas las sociedades cotizadas que forman parte del IBEX, 35 disponen de sitio web, mostrándose en éste su informe financiero anual. Con la promulgación de la Ley dirigida a reforzar la transparencia de las sociedades anónimas cotizadas, en julio de 2003 se ha introducido la obligación legal a todas las sociedades cotizadas de que las mismas dispongan de una página web, a efectos de difusión de información relevante, de la que naturalmente forma parte la información contable-financiera a que nos venimos refiriendo.

A pesar del escaso tiempo transcurrido desde el comienzo de esta nueva vía de comunicación de la información financiera -los primeros estudios empíricos relativos a la divulgación financiera en Internet comienzan a partir de 1996 (Gandía, 2001)- lo cierto es que la publicación de estados financieros digitales en la web ha alcanzado un cierto grado de madurez, habiendo sido utilizados como mecanismos de reproducción archivos pdf., hojas de cálculo y archivos html ${ }^{10}$. Todos estos mecanismos tienen en común que su diseño ha sido realizado pensando en un único tipo de usuario: los seres humanos.

Pero la cadena de suministro de información financiera empresarial no finaliza con la difusión de información que realizan las propias compañías emisoras de la misma. Como reiteradamente viene siendo puesto de manifiesto (Di Piazza et al., 2002,AECA, 2003), tras esta fase quedan todavía otras etapas adicionales, como son aquellas que implican a los distribuidores de información (vendedores de información empresarial), a los analistas independientes (profesionales del asesoramiento financiero) y a los inversores y otros terceros interesados (consumidores últimos de la información empresarial), aparte de las implicaciones que tiene sobre todo el proceso, las instituciones emisoras de normas de contabilidad y de auditoría y las agencias gubernamentales supervisoras.

Para el eficiente desempeño de las tareas a cargo de todos los anteriores se precisan otros mecanismos de reproducción de los estados financieros digitales, que permitan el paso de los mismos de un programa de ordenador a otro, de manera tal que los usuarios intermedios puedan trabajar con sus datos, incorporando los mismos de forma automática a sus modelos de análisis, evitando así seguros costes y posibles errores. Ninguno de los mecanismos de reproducción anteriormente citados permite un uso eficiente de nuevas aplicaciones de software, como hemos dicho, éstas no eran su destinatario, por tanto resulta necesario contar con una nueva herramienta que permita asegurar la transmisión fluida

10 El lenguaje HTML (Hiper Test Markup Language) es el medio más utilizado para la comunicación de información en la web, pero solamente permite describir cómo se distribuye y qué formato tiene la información que se muestra en pantalla, no especificando nada sobre el significado de los datos que se envían. 
ACTIVOS | REVISTA DE LA FACULTAD DE CONTADURÍA PÚBLICA

y segura de datos a través de la cadena de suministro de información empresarial a la que nos venimos refiriendo.

Para ello ha sido concebido un nuevo lenguaje, denominado "Extensible Business Reporting Language" (XBRL) ${ }^{11}$, que facilita el intercambio automático de información entre aplicaciones de software, utilizando un conjunto de taxonomías que definen un marco para el intercambio de datos, de acuerdo con unas determinadas normas de información contablefinanciera. Así, las Normas Internacionales de Contabilidad (NIC/NIIF) permiten definir una taxonomía XBRL, lo que significa que cada elemento de un estado financiero formulado con arreglo a las NIC/NIIF, debe ser identificado y etiquetado independientemente con una denominación precisa, la cual podría ser reconocida por distintas aplicaciones de software, que reproducirían el dato y la denominación del elemento correspondiente en el idioma de trabajo utilizado por el usuario de la aplicación. Teniendo en cuenta que las mencionadas Normas Internacionales de Contabilidad han sido asumidas por la Unión Europea, afectando a las sociedades cotizadas a partir del ejercicio 2005, la presentación de los informes financieros en lenguaje XBRL a partir de dicha fecha, supondría una muy importante mejora efectiva de la cadena de suministro de información empresarial en Europa.

Por supuesto, la utilización de otros principios de contabilidad generalmente aceptados en distintos ámbitos -los US GAAP en los Estados Unidos o los diferentes principios contables nacionales en otros países- exigen sus correspondientes taxonomías XBRL, lo que pone de manifiesto, una vez más, la importancia de llegar a disponer de un único lenguaje contable global. En tanto llega éste, XBRL está ya haciendo posible la comparación entre diferentes taxonomías, definiendo las diferencias entre sus distintos elementos a efectos de permitir la conversión de un documento contable desde una taxonomía a otra.

En resumen, la aplicación de las nuevas tecnologías de la información y la comunicación a la difusión de la información contable-financiera empresarial, pese a haberse iniciado hace corto tiempo, está en constante proceso de cambio y mejora, habiendo quedado claro que con la publicación del informe financiero por parte de las compañías no termina la cadena de suministro de información, ésta representa sólo un escalón más, un input para los siguientes agentes que forman parte de dicha cadena de suministro. Con la utilización del lenguaje XBRL todos los mencionados agentes resultarán beneficiados, las propias compañías, los distribuidores de datos, los analistas, los reguladores y, por supuesto, los

11 XBRL es una adaptación del lenguaje XML (Extended Markup Language), dirigida a regular la transmisión de información contable, principalmente la contenida en los estados financieros elaborados por las empresas. 
usuarios finales, así como también las empresas de software implicadas en el proceso. Es muy posible que la práctica que ahora comienzan a ejercitar las empresas más punteras vaya incorporando con rapidez nuevos practicantes e, inclusive, que los reguladores no se limiten a requerir la publicación de la información financiera en la web, sino que también maticen sus exigencias con respecto a la utilización de un lenguaje eficiente como el XBRL para la transmisión automática de datos.

\section{Gestión contable de los beneficios}

Los escándalos financieros de ENRON y Worldcom en los Estados Unidos han situado la utilización de prácticas contables de conveniencia, mediante las que presentan unas cifras de beneficios que no corresponden con los realmente obtenidos por las empresas. Es cierto que siempre puede existir alguna flexibilidad a la hora de aplicar los principios y normas de contabilidad, generalmente aceptados, pero otra cosa muy distinta es la utilización de prácticas contables fraudulentas, como en los casos antedichos, o bien la utilización de procedimientos contables para "gestionar los beneficios". Esta gestión, alteración, alisamiento o manipulación de los beneficios (Earnings Management) mediante procedimientos contables, ha venido siendo objeto de atención en la literatura académica, a partir de la formulación en la década de los setenta por Watts y Zimmerman (1986) de la "Teoría positiva de la Contabilidad", convirtiéndose en los últimos años en una de las principales líneas de investigación, teniendo en cuenta su trascendencia para los distintos usuarios de la información contable ${ }^{12}$.

Diversos autores han tratado de definir este proceso de gestión contable de beneficios: "intervención deliberada en el proceso de elaboración de la información financiera con el propósito de obtener algún beneficio propio" (Schipper, 1989, p. 92); "selección de técnicas por parte de la gerencia de la empresa para obtener un nivel de beneficios deseado, sirviéndose de la flexibilidad permitida por los principios de contabilidad generalmente aceptados" (Apellániz \& Labrador, 1995, p. 15); la gestión de beneficios tiene lugar cuando los directivos estructuran las transacciones para alterar la información contable-financiera, con el ánimo de conducir a conclusiones erróneas sobre los resultados de la empresa a los usuarios de dicha información o influir en los resultados de los contratos que dependen de las cifras contables (Healy \& Wahlen, 1999).

Esta actuación pro-activa de la gerencia para alcanzar por medios contables los beneficios apetecidos, ha puesto en marcha un amplio número de investigaciones, tratando de

Monterrey (1998) explica la razón de ser y la génesis de este nuevo enfoque de la investigación contable. 
ACTIVOS | REVISTA DE LA FACULTAD DE CONTADURÍA PÚBLICA

revelar a través de las mismas las motivaciones que inducen la actuación de la gerencia en la dirección apuntada, las prácticas contables aplicadas para "gestionar" el beneficio y los métodos de investigación mediante los que detectan la utilización de tales prácticas. La extensión internacional de este fenómeno ha dado lugar a que las investigaciones empíricas realizadas no se refieran exclusivamente a las empresas del país donde se inició esta corriente, Estados Unidos, por el contrario, existen también numerosos estudios relativos a empresas europeas, algunos de ellos referidos a la realidad española.

Paralelamente, también han alcanzado gran importancia las medidas de refuerzo y control que deben ser aplicadas en los sistemas de información contable-financiera para evitar el exceso de "gestión de beneficios", tema éste que ha sido incluido dentro del conjunto de las denominadas prácticas de buen gobierno de la empresa, que será tratado en el siguiente apartado. En los epígrafes que siguen nos referiremos sucintamente a las motivaciones y a las prácticas contables de "gestión de beneficios".

\section{Motivaciones de la "gestión de beneficios"}

Las motivaciones de la dirección para la gestión de beneficios estudiadas por los investigadores fueron inicialmente las de índole contractual y las de naturaleza política y gubernamental y, posteriormente, en la segunda mitad de los noventa del pasado siglo, quedaron puestas de manifiesto las motivaciones relativas a la valoración de la empresa por el mercado. A renglón seguido nos referiremos a unas y otras.

La relación entre ciertas cláusulas de los contratos de deuda suscritos por las empresas y determinadas magnitudes contables - por lo general el ratio de endeudamiento ${ }^{13}$ - incentiva a la dirección de las compañías para acomodar las cifras contables a los supuestos más beneficiosos de los contratos, evitando así mayores tipos de interés o cualquier otro género de penalización; la ocurrencia de lo anterior es más probable en compañías con elevados niveles de endeudamiento, dado su mayor perfil de riesgo.

Los contratos de remuneración de los directivos, dirigidos a alinear los intereses de éstos con los de los accionistas, suelen estar también basados en magnitudes contables - por lo general en la cifra de beneficios- lo que incentiva a los citados directivos para desarrollar políticas contables que den lugar a mayores importes de beneficios, los cuales redundan ventajosamente en sus propias economías. Pero, más allá de los contratos, los directivos

Relación por cociente entre fondos ajenos y fondos propios. 
pueden sentirse presionados por su reputación y por su deseo de continuidad en la dirección de la empresa, lo que también puede moverles a adoptar una determinada estrategia contable; por ejemplo, ha sido estudiado y documentado (De Angelo, 1988) que durante el proceso de selección los directivos intentan ofrecer una buena imagen de la empresa, pero una vez incorporados al cargo empeoran dicha imagen con métodos contables, culpando a los anteriores directivos de una mala gestión, despejando así el camino para la contabilización de beneficios en ejercicios venideros.

Las relaciones entre empresas y administraciones gubernamentales derivadas de procesos políticos tales como: regulaciones sectoriales, defensa de la competencia, leyes fiscales, etc., toman también como referencia las cifras contables para determinar la posición económica de la empresa. Debido a esto, las empresas pueden verse incentivadas para "gestionar" dichas cifras contables en aras de ofrecer una imagen que reduzca los costes derivados del proceso político. Esto ocurre, por ejemplo, con las empresas de gran tamaño, que aplican políticas contables conservadoras para presentar menores beneficios cuando sus elevados resultados pudieran ser considerados como rentas de monopolio (Zmijeswski \& Hagerman, 1981).

Otros estudios realizados se refieren a diversos sectores tales como el petrolífero, televisión por cable, medico-hospitalario, eléctrico y bancario; en España estos dos últimos sectores han sido objeto de sendas investigaciones, relativas a la regulación de precios en el sector eléctrico (Gill e Illueca, 2003) y a los efectos de la regulación contable en la banca (Apellániz, 1991; Arcas, 1993).

A las anteriores motivaciones de la dirección de las compañías para "gestionar" los beneficios, la investigación vino a añadir otra de gran trascendencia: la influencia de las cifras contables en la valoración de la empresa por los mercados de valores. Distintos estudios han venido a demostrar que aquellas empresas que tenían resultados positivos, un crecimiento sostenido de la cifra de resultados e importes de beneficios iguales o superiores a las predicciones de los analistas financieros, eran mejor valoradas por el mercado que el resto. Las mencionadas predicciones han actuado como puntos de referencia (Earnings benchmarks), convirtiéndose en uno de los mayores incentivos para el desarrollo de prácticas de "gestión de beneficios".

Las fuertes presiones sentidas por empresas como ENRON o Wolrdcom para cumplir con las predicciones de analistas e inversores les llevaron a desarrollar prácticas de manipulación del resultado tan agresivas que finalmente se convirtieron en fraudulentas (García \& Gisbert, 2003, García, Gill \& Gisbert, 2003). También las empresas españolas tienden 
ACTIVOS | REVISTA DE LA FACULTAD DE CONTADURÍA PÚBLICA

a aumentar sus resultados para evitar el reconocimiento de pérdidas y descensos de sus beneficios, según ponen de manifiesto Gill y Alcarria (2003) y Giner y Gallen (2003).

Otros estudios han analizado la existencia de prácticas de "gestión de beneficios" con ocasión de ciertos acontecimientos singulares, como por ejemplo en la salida inicial a la Bolsa o en posteriores emisiones, dada la asimetría informativa existente entre los directivos y los futuros inversores.

Otras operaciones estudiadas han sido las de compra de empresas por sus propios directivos, quienes pueden influir en los resultados contables de los ejercicios anteriores a la operación de compra, así como también los casos de fusiones de empresas en los que se produce un intercambio de acciones, cuya relación de canje ha podido verse influida por los mayores beneficios contabilizados por la sociedad absorbente en fechas previas a la de la fusión. Gabás, Apellániz (1999) han investigado sobre los factores determinantes de la elección del método contable en la fusión de empresas por parte de empresas españolas y su influencia sobre los beneficios presentados por la nueva entidad fusionada.

\section{Prácticas contables y financieras}

Las prácticas contables utilizadas para la "gestión de beneficios" consisten básicamente en desplazar alguno de los componentes de las corrientes de ingresos y gastos de un ejercicio a otro. Algunos autores han realizado importantes esfuerzos de sistematización de las operaciones involucradas y de su respectivo grado de importancia relativa en la realidad fáctica investigada (Nelson, Elliot \& Tarpley, 2003), aquí no entraremos en tanto detalle, limitándonos a hacer referencia a las partidas más corrientemente utilizadas en la gestión contable de beneficios.

Los directivos prefieren "gestionar el beneficio" haciendo uso de aquellas prácticas que resultan menos visibles y menos costosas. En relación con la visibilidad, cabe señalar que unas partidas son seguidas más de cerca que otras por los analistas financieros e inversores, dada su relevancia en el contexto de la empresa correspondiente; además, los principios de contabilidad generalmente aceptados exigen mayores niveles de revelación de información en unos casos que en otros, lo que lleva a preferir, a efectos de "gestión de beneficios", aquellos que resulten menos visibles.

Por lo que se refiere al coste de utilizar una práctica u otra, éste es función del riesgo asumido por la empresa frente a los inversores y las autoridades oficiales, cuando éstos 
pueden penalizar a la empresa por la deficiente práctica utilizada; lógicamente las prácticas fraudulentas serán las más costosas, dadas las secuelas que puede traer el descubrimiento de las mismas, por tanto, intentarán ser evitadas, aunque la realidad demuestra que cuando el uso intensivo de otras opciones menos costosas no permite alcanzar el nivel de beneficios deseado, puede caerse en la tentación de traspasar la frontera de la gestión para adentrarse en el terreno de la auténtica manipulación de beneficios.

Las prácticas contables más utilizadas para la gestión de beneficios son los ajustes por devengo y las dotaciones a las provisiones por operaciones de tráfico (morosidad y otros), por ser éstas las menos visibles y menos costosas. Los cambios en los métodos de amortización resultan más visibles, aunque también son objeto de utilización. Igualmente el cálculo del efecto impositivo (impuestos anticipados y diferidos) ha sido detectado como una partida utilizada en este tipo de prácticas de gestión de beneficios. Además, con ocasión de operaciones de reestructuración, la imputación a resultados de los correspondientes costes también ha sido objeto de frecuente utilización, inclusive algunos de tales costes han sido directamente cargados contra reservas, evitando así su incidencia sobre la cifra de beneficios.

Más allá de los meros cambios en los registros contables a que se refieren las operaciones anteriores, se encuentran ciertas decisiones financieras, las cuales vienen condicionadas por el efecto que las mismas tienen sobre la determinación del beneficio. El momento en que se decide vender un activo lleva consigo el reconocimiento en una fecha $\mathrm{u}$ otra del resultado (ganancia o pérdida) correspondiente, lo que incentivará a su realización inmediata o a su aplazamiento ${ }^{14}$; lo anterior consiste en aprovechar un ejercicio con escaso resultado operativo para realizar ganancias con la venta de ciertos activos cuyo valor contable es inferior al del mercado.

Otra importante decisión estratégica condicionada por las normas contables es la relativa a la selección de los proyectos de $\mathrm{I}+\mathrm{D}$ en los que invertir, dada la repercusión inmediata de los costes correspondientes en los resultados del ejercicio, que puede llevar a inclinarse no por los proyectos mejores sino por los menos costosos o, simplemente, por posponer la inversión ante la perspectiva de ver reducidos los resultados del ejercicio; los directivos pueden otorgar preferencia a la perspectiva de corto plazo, aunque ésta no coincida con la decisión óptima para la empresa.

14 Una de las dificultades de introducción del criterio del valor razonable procede de la eliminación de la discrecionalidad temporal de reconocimiento del beneficio por venta de instrumentos financieros, al obligar a contabilizar dicho beneficio, año tras año, según el valor de mercado, con independencia de que se vendan o no los activos correspondientes. 
ACTIVOS | REVISTA DE LA FACULTAD DE CONTADURÍA PÚBLICA

\section{A modo de conclusión}

La información contable-financiera de las sociedades ha pasado a ser considerada como un bien jurídicamente protegible, dada la utilidad de la misma para un amplio conjunto de usuarios, entre los que se incluyen los accionistas, los empleados, los acreedores y otros terceros interesados en la marcha de la empresa. Los inversores, sean grandes $o$ pequeños, reclaman a las empresas una mayor transparencia y una mejor información, esforzándose por ganar influencia sobre las sociedades de las que son accionistas. Las autoridades de la Unión Europea que establecieron como objetivo la libre circulación de capitales en el mercado interior, consideraron que para el cumplimiento de dicho objetivo, la protección de los inversores y el mantenimiento de la confianza de los mercados financieros era necesario profundizar en el proceso de armonización contable europea, yendo más allá de las directivas de derecho de sociedades y adoptando las Normas Internacionales de Contabilidad o Normas Internacionales de Información Financiera, de manera tal que los estados financieros consolidados de las sociedades cotizadas en bolsa resulten comparables.

Pero, al mismo tiempo que el proceso de regulación y armonización contable progresa en la dirección que acabamos de apuntar, han venido produciéndose importantes cambios tecnológicos, económicos y sociales que afectan profundamente al contenido, a la difusión y a la estrategia de preparación de la información financiera. El desarrollo de la economía basada en el conocimiento ha puesto de relieve la importancia de los intangibles como factores generadores de valor, la información financiera de las empresas no puede seguir anclada en unos principios y normas contables procedentes de los tiempos de una economía manufacturera, tiene que incorporar nuevos criterios que permitan visualizar la importancia de los nuevos factores que crean valor, ya sea utilizando los clásicos instrumentos de medición y reconocimiento en balance y resultados, ya mediante sistemas de indicadores o revelación de información en memoria; los mercados serán más eficientes en su asignación de recursos si disponen de información sobre los factores que en la actualidad están creando valor.

La comunicación de la información financiera por medio de Internet está suponiendo una auténtica revolución que no acaba sino de empezar. Aparte de que este tipo de difusión sea oportuna, interactiva, cómoda, económica y frecuente, las posibilidades de reutilización de dicha información por los usuarios de la misma están ampliándose enormemente mediante el nuevo lenguaje XBRL, el cual facilita el intercambio automático de información entre aplicaciones de software. Esta nueva herramienta permitirá que las tareas subsiguientes 
de supervisores, reguladores, analistas e inversores puedan ser realizadas de forma más rápida y segura.

Los escándalos de ENRON, Worldcom y otros, han situado en un primer plano de actualidad las prácticas contables de gestión de beneficios realizadas por la dirección de las sociedades. Es cierto que, cuando se habla de gestión de beneficios, se está pensando en un movimiento estratégico dentro de los límites de flexibilidad permitidos por los principios de contabilidad generalmente aceptados, pero no es menos cierto que unos pasos pueden ir seguidos de otros, pasando de la gestión a la manipulación y después al fraude.

La investigación contable inmersa en la línea de la teoría positiva de la contabilidad viene poniendo de manifiesto las motivaciones de los directivos para la utilización de políticas de gestión de beneficios así como las prácticas contables y financieras utilizadas. No se trata de un fenómeno localizado en unas pocas empresas, existen evidencias de su amplia utilización en Estados Unidos y en Europa, y también en España. Ante la emergencia de los escándalos empresariales antedichos y, teniendo en cuenta lo que la investigación viene revelando, no es de extrañar el incremento de la regulación legal en materias tales como información financiera, auditoría y gobierno de la empresa.

En conclusión, partiendo del reconocimiento de la gran importancia que tiene la armonización internacional de las normas dirigidas a la preparación de la información contablefinanciera de las sociedades, es preciso, al propio tiempo, dar respuesta a los nuevos retos surgidos en relación con el contenido, la comunicación y la estrategia de preparación de la citada información, en aras de preservar la utilidad de la misma para el amplio conjunto de usuarios a quienes va dirigida, dada su condición de bien jurídicamente protegible.

\section{Referencias}

AECA (2003). XBRL un estándar para el intercambio electrónico de información económica y financiera. Madrid: AECA.

Apellániz, P. (1991). Una aproximación empírica al alisamiento de beneficios en la banca española. Revista Española de Financiación y Contabilidad, 66.

Arcas, M. J. (1993). Impacto bursátil de la regulación contable en la banca española: la provisión para insolvencias y riesgo-país. Revista Española de Financiación y Contabilidad, 74 . 
ACTIVOS | REVISTA DE LA FACULTAD DE CONTADURÍA PÚBLICA

Ballester, M., García-Ayuso, M. \& Livnat, J. (2003). Estimating the R\&D Intangible Asset. European Accounting Review, 12, (4).

Blair, M. \& Wallman S. (2001). Unseen Wealth. Washington D. C.: Brookings Institution.

Bonsón, E. (2001). The Role of XBRL in Europe. The International Journal of Digital Accounting Research, 1, (2), pp. 101-110.

Bukh, P., Johansen, M. \& Mouritsen, J. (2002). Multiple Integrated Performance Management Systems: IC And BSC In A Software Company. Singapore Management Review, 24, (3), 21-34.

Cañibano, L., Garcia-Ayuso, M. \& Sánchez, M. (1999). The Value Relevance And Managerial Implications Of Intangibles: a Literature Review. In J.E. Gröjer And H Stolowy (Eds) Classification Of Intangibles. Groupe HEC, Jouy-En Josas, France, 78-126.

Cañibano, L., Garcia-Ayuso, M. \& Sánchez, M. (2000a). Accounting For Intangibles: a Literature Review. Journal Of Accounting Literature, 19, 102-130.

Cañibano, L., Garcia-Ayuso, M. \& Sánchez, M. (2000b). Shortcomings in the Measurement of Innovation: Implications for Accounting Standards Setting. Journal of Management and Governance, 4, 319-342.

Cañibano, L. \& Sánchez, M. (2003). Measurement, Management and Reporting on Intangibles. State Of The Art. Paper presented at The American Accounting Association Annual Meeting. Hawaii.

Cañibano, L.; Sanchez, M., Garcia-Ayuso, M. \& Chaminade, M. (2002). Meritum Guidelines for Managing and Reporting on Intangibles. Madrid: Fundación Vodafon.

Carreira, F. (2003). O Relato Financiero e a internet: Estudo comparativo das empresas que integraram os indices PSI 20 e IBEX 35 entre julio de 2000 e dezembro de 2001. (tesis doctoral). Universidad Autónoma de Madrid.

Chaminade, C. \& Johanson, U. (2003). Can Guidelines for Intellectual Capital Management and Reporting be considered without addressing cultural differences? Journal of Intellectual Capital (Special Issue on The Transparent Enterprise. The Value of Intangibles), 4, (4), 528-542. 
Chaminade, C. \& Cañibano, L. (2003). La gestión de los intangibles y el aprendizaje empresarial. En J. M. Casado. “Desaprendizaje organizativo”, 181-201. Barcelona: Ariel.

Danish Agency for Trade and Industry (DATRI) (2002). A Guideline for Intellectual Capital Statements- A Key to Knowledge Management. Copenhagen: DATRI.

Danish Ministry of Science and Technology (2003a). Intellectual Capital Statements The New Guideline. Danish Ministry of Science and Technology. Copenhagen.

Danish Ministry of Science and Technology (2003b). Analysing Intellectual Capital Statements. Danish Ministry of Science and Technology. Copenhagen.

DeAngelo, L. E. (1988). Managerial competition, information costs, and corporate governance: the use of accounting performance measures in proxy contests. Journal of Accounting and Economics, 10, 3-36.

DiPiazza, S. \& Eccles, R. (2002). Recuperar la confianza: el futuro de la información corporativa. Madrid : FT Prentice Hall.

Financial Accounting Standards Board (FASB). (2001). Statement of Financial Accounting Standards no 142: Goodwill and Other Intangible Assets. FASB. Norwalk, CT.

Financial Accounting Standards Board (FASB). (2003). Disclosure about Intangible Assets www.fasb.org/project/intangibles.shtml.

Gabás, F., Apellániz, F. \& Apellániz, P. (1999). Factores determinantes de la elección del método contable en las fusiones de empresas. Revista Española de financiación y Contabilidad, 100, 111-149.

Gandía, J. L. (2001). La divulgación de información financiera en la era digital. Madrid: AECA.

Gandía,J.L. (2002).La divulgación de información financiera sobre intangibles en Internet: evidencia internacional. Revista Española de Financiación y Contabilidad, 113, 767-802.

García-Ayuso, M. (2003). Factors explaining the inefficient valuation of intangibles. Accounting, Auditing and Accountability Journal, 1. 
ACTIVOS | REVISTA DE LA FACULTAD DE CONTADURÍA PÚBLICA

García, B. \& Gisbert,A. (2003). Earnings Management:A literature review. Revista Española de Financiación y Contabilidad, 115, 311-323.

García, B.; Gill, B. \& Gisbert,A.(2003).La literatura sobre la manipulación del resultado: estado del arte y perspectivas. Working paper, Lancaster University, Universitat Jaume I y Universidad Autónoma de Madrid. XII Congreso AECA, Cádiz 29 de septiembre, 1 de octubre de 2003.

García, J. \& Mora,A. (2003a). La incorporación asimétrica de noticias al resultado contable en un contexto europeo: evidencia empírica. Revista Española de Financiación y Contabilidad, (Aceptado para publicación).

García, J. \& Mora, A. (2003b). On The Persistent Understatement of Shareholders'Equity Around Europe. Revista Española de Financiación y Contabilidad, 115.

Gill, B. \& Alcarria, J. (2003). Analysis and diagnosis of income smoothing in Spain. European Accounting Review, 12, 443-463.

Gill, B. \& Illueca, M.(2003). Regulación de precios y prácticas de Earnings Management: Evidencia empírica en el sector eléctrico español. Estudios Financieros, 247, 155-192.

Giner, B. \& Gallen, M. (2003). La alteración del resultado para evitar pérdidas y descensos: una evidencia empírica. (Documento de trabajo), Universidad de Valencia.

Giner, B. \& Pardo, F. (2002). Is Goodwill Amortization Value Relevant for Share Prices? A European Perspective, (Documento de trabajo). Universidad de Valencia.

Giner, B. \& Rees, W. (2001). On The Asymmetric Recognition of Good And Bad News in France, Germany And The United Kingdom. Journal Of Business, Finance and Accounting, 28, (9-10), 1285-1331.

Gonzalo, J. (2002a). Informe sobre la situación actual de la contabilidad en España y líneas básicas para abordar su reforma. Libro Blanco para la reforma de la contabilidad en España. Madrid: Ministerio de Economía, ICAC.

Guthrie, J.; Petty, R. \& Johanson, U. (2001). Sunrise in The Knowledge Economy: Managing, Measuring and Reporting Intellectual Capital. Accounting, Auditing \& Accountability Journal, 14, (4). 
Healy, P. \& Wahlen, J. (1999). A review of the earnings management literature and its implication for standard setting. Accounting Horizons, 13.

Hendriksen, E. \& Van Breda, M. (1992). Accounting Theory. Irwin, Burr Ridge.

Holland, J. \& Johanson, U. (2003). Value Relevant Information on Corporate Intangibles - Creation, Use, and Barriers in Capital Markets - Between a Rock and a Hard Place. Journal of Intellectual Capital, 4, (4), 465-486.

Johanson, U. (2000). Human Resource Accounting. Putting a Price on Human Assets. Brewster, C and H. H. Larsen (Eds.) Human Resource Management in Nothern Europe. Blackwell. 170-195.

Johanson, U. (2002). Management of Knowledge and Intellectual Capital. Special Issue of Singapore Management Review, 24, (3).

Johanson, U., Eklöv, G., Holmgren, M., \& Mårtensson, M. (1999). Human Resource Costing and Accounting Versus the BSC. A Literature Survey of Experience With the Concepts. A Report to OECD. OECD. Http://Www.Oecd.Org/Dsti/Sti/Industry/ Indcomp/Act/Ams-Conf/Symposium.Htm.

Johanson, U. Mårtensson, M. \& Skoog, M. (2001a). Measuring to Understand Intangible Performance Drivers. European Accounting Review, 10, (3).

Lev, B. (2001). Intangibles: Management, Measurement and Reporting. Brookings Institutions. Washington D. C. Versión española (2003) Intangibles: Medición, Gestión e Información. Deusto, Barcelona.

Lev, B. \& Sougiannis, T. (1996). The capitalization, amortization and value relevance of $\mathrm{R}$ \& D. Journal of Accounting and Economics, 21, 107-138.

Lev, B. \& Zarowin, P. (1998). The Boundaries of Financial Reporting and how to extend them. Journal of Accounting Research. Supplement 37, 353-385.

Monterrey, J. (1998). Un recorrido por la contabilidad positiva. Revista Española de Financiación y Contabilidad, 95, 427-467. 
ACTIVOS | REVISTA DE LA FACULTAD DE CONTADURÍA PÚBLICA

Mouritsen, J.; Larsen, T. \& Bukh, P. (2001a). Intellectual Capital and the 'Capable Firm': Narrating, Visualising and Numbering for Managing Knowledge. Accounting, Organisations And Society, (7/8), 715-733.

Mouritsen, J.; Larsen, T. \& Bukh, P. (2001b). Valuing the Future: Intellectual Capital Supplements at Skandia, Accounting, Auditing \& Accountability Journal, 14, (4).

Nelson, M.; Elliot, J. \& Tarpley, R. (2003). How are Earnings Managed? Examples from Auditors. Accounting Horizons. Supplement, 17-35.

Organisation for Economic Co-operation and Development (1997a). Measuring Performance in The Age Of Intangibles. Enterprise Value in The Knowledge Economy. Conference in Helsinki In 1996. OECD and Ernst \& Young, Boston.

Organisation for Economic Co-operation and Development (1997b). Changing Workplace Strategies: Achieving Better Outcomes for Enterprises, Workers and Society. Report on the International Conference in 1996 Organised by the OECD and the Government of Canada.

Organisation for Economic Co-operation and Development (1999). Measuring and Reporting Intellectual Capital: Experience, Issues and Prospects. Amsterdam, June. Http://Www.Oecd.Org/Dsti/Sti/Industry/Indcomp/Act/ Amsconf /Symposium.Htm.

Ohlson, J. (1995). Earnings, Book Values, and Dividens in Equity Valuation. Contemporary Accounting Research, 11, (2), 661-687.

Petty, R. \& Guthrie, J. (2000). Intellectual Capital Literature Review. Journal of Intellectual Capital, 1, (2), 155-176.

Roberts, H. (2002). Guidelines for Additional Information of Value Creation. The NFF Guideline. The International Conference "The Transparent Enterprise. The Value of Intangibles". Madrid, november 2002.

Roberts, H. \& Chaminade, C. (2003). What it means and what it does: a comparative analysis of implementing Intellectual Capital in Norway and Spain. The European Accounting Review, 12, (4), 733-751. 
Sánchez, P., Chaminade, C. \& Olea, M. (2000). Management of Intangibles: an attempt to build a theory. Journal of Intellectual Capital, 1, (4), 312-327.

Shipper, K. (1989). Commentary on Earnings management. Accounting Horizons, 3, 91-102.

Unión Europea (UE) (2003). Study on The Measurement of Intangible Assets and Associated Accounting Practices. Disponible. Http://Europa.Eu.Int/Comm/ Enterprise/Services/ Business_Services/Papers.Htm.

Vickery, G. (1999). Accounting for Intangibles: Issues and Prospects. In Buiges, P., Jacquemin, A., Marchipont, F. Intangibles And Competitiveness: An Empirical Approach. Edward Elgar, Aldershot.

Wallman, S. (1995). The Future of Accounting and Disclosure in an Evolving World: the need for a dramatic change. Accounting Horizons, 9, (3), 81-91.

Wallman, S. (1996). The Future of Accounting and Financial Reporting: part II: The Colorized Approach. Accounting Horizons, 10, (2), 138-148.

Watts, R. \& Zimmerman, J. (1986). Positive Accounting Theory. Prentice Hall, Homewood, Ill.

Zmijewski, M. \& Hagerman, R. (1981). An income strategy approach to the positive theory of accounting standard setting choice. Journal of Accounting and Economics, 3, 129-149. 